\title{
The red-sky enigma over Svalbard in December 2002
}

\author{
F. Sigernes ${ }^{1}$, N. Lloyd ${ }^{2}$, D. A. Lorentzen ${ }^{1}$, R. Neuber ${ }^{3}$, U.-P. Hoppe ${ }^{4}$, D. Degenstein ${ }^{2}$, N. Shumilov ${ }^{1,5,{ }^{*}}$, J. Moen ${ }^{1,6}$, \\ Y. Gjessing ${ }^{1,7}$, O. Havnes ${ }^{1,5}$, A. Skartveit ${ }^{7}$, E. Raustein ${ }^{7, *}$, J. B. Ørbæk ${ }^{8}$, and C. S. Deehr ${ }^{9}$ \\ ${ }^{1}$ The University Centre in Svalbard (UNIS), N-9171 Longyearbyen, Norway \\ ${ }^{2}$ ISAS, University of Saskatchewan, Saskatoon, Canada \\ ${ }^{3}$ Alfred Wegener Institute for Polar and Marine Research, Potsdam, Germany \\ ${ }^{4}$ Norwegian Defence Research Establishment, Kjeller, Norway \\ ${ }^{5}$ The Auroral Observatory, University of Troms $\varnothing$, Norway \\ ${ }^{6}$ Department of Physics, University of Oslo, Oslo, Norway \\ ${ }^{7}$ Geophysical Institute, University of Bergen, Bergen, Norway \\ ${ }^{8}$ The Norwegian Polar Institute, Longyearbyen, Svalbard, Norway \\ ${ }^{9}$ Geophysical Institute, University of Alaska, Fairbanks, USA \\ *Deceased
}

Received: 31 August 2004 - Revised: 13 January 2005 - Accepted: 17 January 2005 - Published: 27 July 2005

Part of Special Issue "Atmospheric studies by optical methods"

\begin{abstract}
On 6 December 2002, during winter darkness, an extraordinary event occurred in the sky, as viewed from Longyearbyen $\left(78^{\circ} \mathrm{N}, 15^{\circ} \mathrm{E}\right)$, Svalbard, Norway. At 07:30 UT the southeast sky was surprisingly lit up in a deep red colour. The light increased in intensity and spread out across the sky, and at 10:00 UT the illumination was observed to reach the zenith. The event died out at about 12:30 UT. Spectral measurements from the Auroral Station in Adventdalen confirm that the light was scattered sunlight. Even though the Sun was between 11.8 and $14.6 \mathrm{deg}$ below the horizon during the event, the measured intensities of scattered light on the southern horizon from the scanning photometers coincided with the rise and setting of the Sun. Calculations of actual heights, including refraction and atmospheric screening, indicate that the event most likely was scattered solar light from a target below the horizon. This is also confirmed by the OSIRIS instrument on board the Odin satellite. The deduced height profile indicates that the scattering target is located $18-23 \mathrm{~km}$ up in the stratosphere at a latitude close to $73-75^{\circ} \mathrm{N}$, southeast of Longyearbyen. The temperatures in this region were found to be low enough for Polar Stratospheric Clouds (PSC) to be formed. The target was also identified as PSC by the LIDAR systems at the Koldewey Station in Ny-Ålesund $\left(79^{\circ} \mathrm{N}, 12^{\circ} \mathrm{E}\right)$. The event was most likely caused by solar illuminated type II Polar Stratospheric Clouds that scattered light towards Svalbard. Two types of scenarios are presented to explain how light is scattered.
\end{abstract}

Correspondence to: F. Sigernes

(freds@unis.no)
Keywords. Atmospheric composition and structure (Transmissions and scattering of radiation; Middle atmospherecomposition and chemistry; Instruments and techniques) History of geophysics (Atmospheric Sciences; The red-sky phenomena)

\section{Introduction}

The Auroral Station in Adventdalen, Svalbard $\left(78^{\circ} \mathrm{N}\right.$, $15^{\circ} \mathrm{E}$ ), close to the town of Longyearbyen (LYR), is a multiinstrument platform for studies of dayside aurora and other high latitude optical phenomena. It is more or less completely dark during the day for more than 2 months in the middle of the winter. Near winter solstice the Sun is at least $10 \mathrm{deg}$ below the horizon at noon. Hence, it should be almost completely dark in the daytime at Longyearbyen during the months of December and January. But, to our surprise, on 6 December 2002, the southeast sky turned deep red from about 07:30 to 12:30 UT. The scattered light from the event turned the polar night into day. In fact, the light was so intense that the whole valley of Adventdalen was visible to the human eye - an effect that is usually not seen at this time of the year except in the light of the full moon.

The whole event caused great public attention. Questions about its origin were very soon directed to the Auroral Station. In the early stages of the event, it was even speculated that a nuclear bomb explosion must have taken place. The Governor of Svalbard was on the alert and the local newspaper made headlines in Norway with the article titled "A 


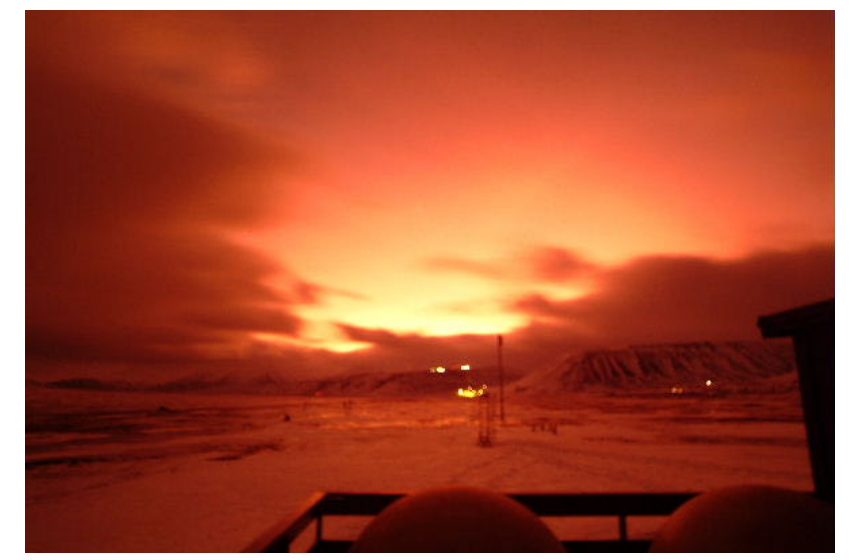

Fig. 1. Photograph of the event taken from the Auroral Station in Adventdalen, Svalbard, Norway, at 08:42:41 UT on 6 December 2002. The exposure time is $20 \mathrm{~s}$ at F/2.8, using a $14-\mathrm{mm}$ lens with a Fujifilm S2Pro camera body. The sensitivity is ISO 200.

mysterious phenomena" (Pedersen, 2002). It is safe to say that during the morning hours of 6 December 2002, we were first scared by the event. Later on, this feeling turned into curiosity.

At the time of the event The Auroral Station in Adventdalen was fully operative with all its instruments, which were set up to study optical signatures of the dayside aurora. A remote controlled spectrometer located in Ny-Ålesund (NYA; $79^{\circ} \mathrm{N}, 12^{\circ} \mathrm{E}$ ) was also operative. Ny-Ålesund is $118 \mathrm{~km}$ north of Longyearbyen. Fortunately, data from the OSIRIS instrument on board the Odin satellite and data from the LIDARS at the Koldewey Station in Ny-Ålesund are also available. This study presents both the ground-based and the space-borne measurements of the event. Finally, a discussion is given on what might be the cause of the red-sky event.

\section{Ground-based observations}

The ground-based data set consists of photographs, photometer scans along the magnetic meridian, wavelength spectra obtained in the zenith from both Longyearbyen and $\mathrm{Ny}$ Ålesund, and multi-wavelength LIDAR height profiles from the Koldewey Station in Ny-Ålesund.

\subsection{Weather conditions}

The sky on 6 December 2002 was partly cloudy over the station with normal wind conditions at ground level. The average wind speed from southeast was $7.2 \mathrm{~m} / \mathrm{s}$ at the 10 $\mathrm{m}$ height level. There was, in other words, a light breeze blowing down the valley (Adventdalen) toward the sea (Isfjorden). The pressure was high (1022 mbar), with a daily average temperature of $+5.4 \mathrm{deg}$ Celsius, which is close to $19 \mathrm{deg}$ Celsius above the daily average temperature for this date measured from 1976 to 1989 . In fact, the whole period from November to December was characterized as being much warmer than the normal conditions. A strong heat advection from the south led to the observed ground temperatures close to +5 deg Celsius.

\subsection{Image gallery}

High resolution digital photographs of the event were obtained by using a Fujifilm FinePixS2Pro camera body with a wide-angle 14-mm focal length len. The original images are $4256 \times 2848$ pixels. Figure 1 shows an unprocessed image taken from the Auroral Station pointing towards the southeast, with the lights of Mine 7 and EISCAT close to the centre of the image. The exposure time was $20 \mathrm{~s}$ at $\mathrm{f} / 2.8$. Note the deep red-coloured snow-covered ground, indicating that strong multiple scattering took place in the troposphere.

\subsection{Meridian scanning photometers}

The Meridian Scanning Photometer (MSP) has 5 channels, where each channel consists of a narrow bandpass filter mounted in a tilting frame. The tilting filter is in front of a 3" telescope with a cooled photomultplier (the detector). These tilting filter photometers are placed in front of a rotating mirror, which scans the sky from north to south along the geo-magnetic meridian. The instrument delivers intensity as a function of elevation angles in the meridian plane in 5 wavelength bands. The field of view is approximately one degree for each channel. The principal wavelengths used in this study are the auroral 5577, 6300 and the $8446 \AA$ emission lines of atomic oxygen. In addition, we use the auroral $4278 \AA$ emission band of molecular nitrogen $\left[\mathrm{N}_{2}^{+} 1 \mathrm{NG}\right]$ and the 4861 A Doppler broadened profile of hydrogen $\left[\mathrm{H}_{\beta}\right]$. The latter emission is normally produced by proton precipitation, producing hydrogen aurora. Each filter has a bandpass of approximately $5 \AA$. The background for each filter is obtained by tilting the filters from peak transmission position at the line emission of interest to an angle that transmits the base wavelength representing the background emissions (BASE). The shift angle is chosen to change the peak wavelength at least 2 times the bandpass. Data is recorded over the meridian every $16 \mathrm{~s}$ ( $2 \times 4$-s-scan line peak; $2 \times 4 \mathrm{~s}$ of BASE).

In Fig. 2 the background or BASE data is shown. The peak values are not used because they are contaminated by the above mentioned auroral emissions. The Near Infra Red (NIR) channel observed the strongest intensities. The intensities decreased with wavelength towards the blue. Note that the data is colour coded in Fig. 2 from black to red in units of Rayleighs, as given by the colour bars to the left. The colour red represents intensities at or above the given maximum numbers. For example, a closer look at 10:00 UT reveals that the $8446 \AA$ channel illumination rises quickly from the horizon up to a maximum at about $168 \mathrm{deg}$, followed by a relatively slow decrease to the zenith $(90 \mathrm{deg})$. The maximum intensity is above $200 \mathrm{kR}$ at $168 \mathrm{deg}$. For the other channels, the maximum intensities are $70,15,5$ and $0.8 \mathrm{kR}$ for the $6300,5577,4861$ and $4278 \AA$ channels, respectively. We find no distinct wavelength difference in the shape of the 


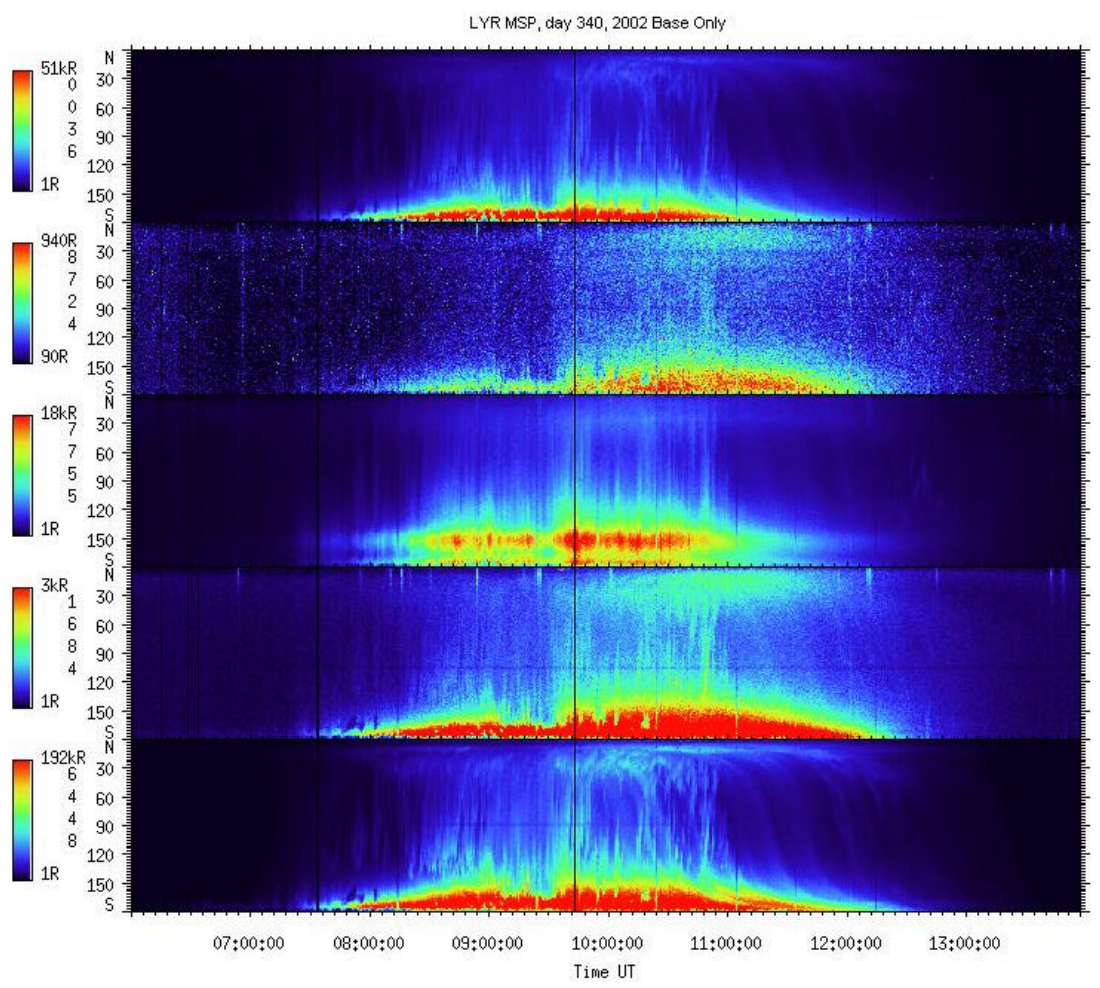

Fig. 2. Meridian Scanning Photometer (MSP) data (BASE Only) from the Auroral Station in Adventdalen, Norway, 06:00-14:00 UT, 6 December 2002. The intensities are indicated as coloured bars to the left for each channel with centre wavelengths $6300,4278,5577,4861$ and $8446 \AA$, respectively. The intensities are in kilo Rayleighs [kR]. South is $180^{\circ}$ in scan angle. Note that solar depression angles were $12.6^{\circ}, 10.7^{\circ}$, and $11.8^{\circ}$ at 08:30 UT, 10:30 UT and 12:30 UT, respectively.

profiles, except for the green channel which has a stronger local maximum at 150 compared to the one at $168 \mathrm{deg}$.

\subsection{Spectrometers}

The Ebert-Fastie spectrometers used in this study were originally designed by Fastie (1952a,b). Two instruments with $1 \mathrm{~m}$ focal length were operated from Longyearbyen (designators Green LYR and Silver LYR), and one $1 / 2 \mathrm{~m}$ focal length instrument in Ny-Ålesund (designator Black Ny $\AA$ ).

The principal components of the instruments are one large $1 \mathrm{~m}$ focal length spherical mirror $(1 / 2 \mathrm{~m}$ focal length for Black NyÅ), one plane reflective diffraction grating and a pair of curved slits. The recorded radiance from the sky is limited by the etendue; the product of the area of the entrance slit and the field of view. Because the intensity of the source is usually low, the etendue is made as large as possible. The image of the entrance slit is reflected by one part of the spherical mirror onto the grating. The second part of the mirror focuses the diffracted light from the grating onto the exit slit. When the grating turns, the image of the entrance slit is scanned across the exit slit. A collector lens transfers the output of the exit slit to the front of a photo multiplier tube. The tube is mounted in a Peltier cooled housing. Signals from the tube are amplified and discriminated before being sent to the computer counting card.
In Fig. 3, the green curves are time-averaged spectra before the event, representing normal conditions with intensities in $\mathrm{R} / \AA \AA$ on the right hand axis. The red curves are the corresponding spectra of our event with intensities in R/Å on the left hand axis. First of all, the normal or background spectra show known familiar features. In panel (A) and (B), the $4861 \AA[\mathrm{H} \beta]$ and the $6563 \AA[\mathrm{H} \alpha]$ Doppler broadened emission lines of hydrogen appear. The source of the emission profiles is proton precipitation, causing the dayside hydrogen aurora. Also, throughout panel (B), (C) and (D) the airglow emission lines of hydroxyl $\mathrm{OH}(6,1), \mathrm{OH}(8,3)$ and $\mathrm{OH}(6,2)$ are dominating the spectrum, especially in the NIR region of the spectrum. The emission lines at $6300 \AA, 6364 \AA$ (Panel B) and $8446 \AA$ (Panel D) are oxygen [OI] lines produced by electron precipitation. Other known auroral lines are the $7320 / 30 \AA$ doublet of oxygen [OII] (Panel C).

In addition, high resolution, ground-based, direct solar spectra (Full Width at Half Maximum; FWHM=0.002 ) have been obtained from the BASS2000 tool provided by the Solar Survey homepage (http://mesola.obspm.fr/). In order to compare with the event (red curves), these spectra have been convolved with the instrumental functions corresponding to the bandpass of our instruments. Furthermore, the spectra are normalized to the intensities of our measurements. The scaling factors are shown in each panel. Comparison of the resulting spectra (the yellow curves in Fig. 3) 


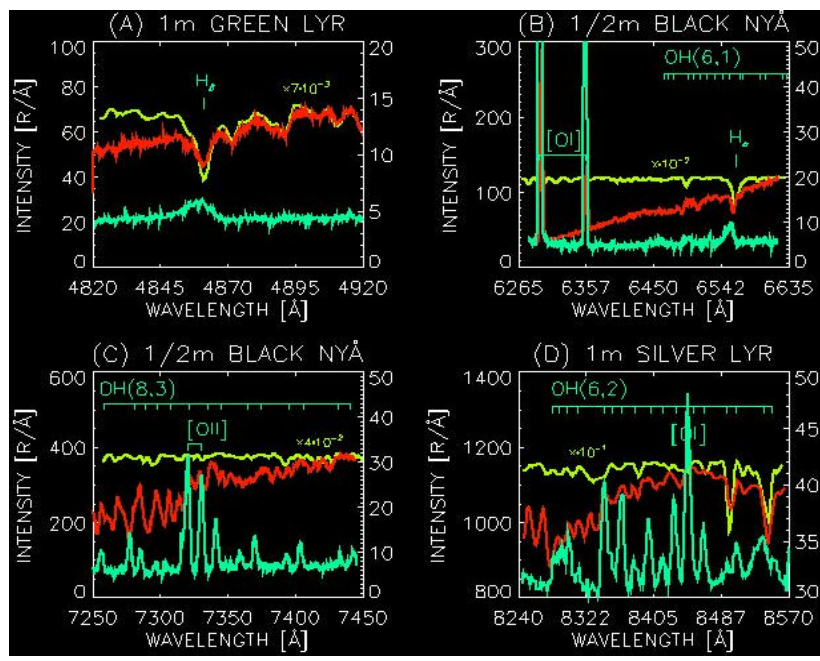

Fig. 3. Spectrometer data of the 6 December 2002 event from the Auroral Station in Adventdalen (Panel A and D) and from the station in Ny-Ålesund (Panel B and C). Green curves are prior to the event, indicating the normal dark sky conditions. Red spectra are from the event itself. The yellow curves are a model spectrum of the sun. The intensities are in Rayleighs/Ångstrøm [R/Å] on both the left and right hand $y$-axis. The right hand axis represents normal conditions (green curve).

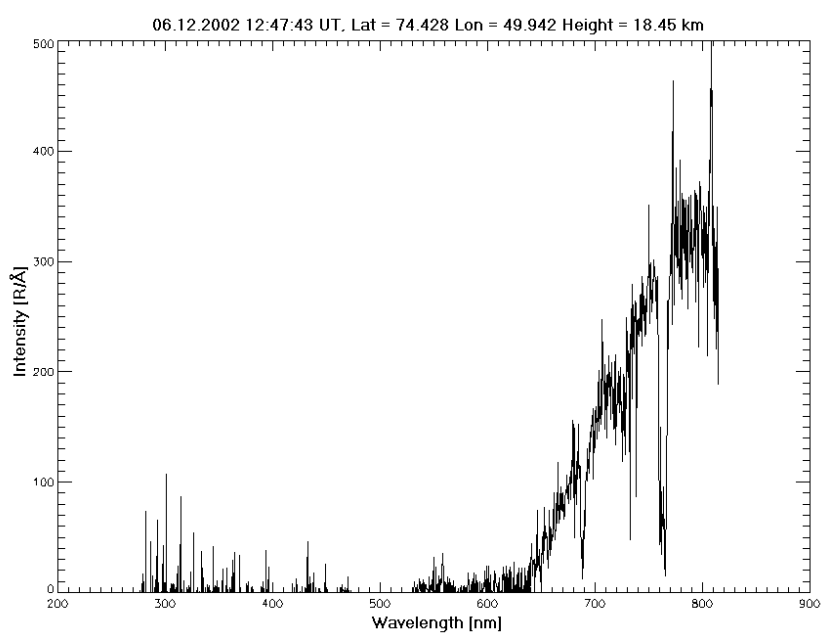

Fig. 4. ODIN / OSIRIS spectrum of the red-sky event at 12:47:43 UT, orbit \#9726,6 December 2002. The target is located at position $\left(74.4^{\circ} \mathrm{N}, 49.9^{\circ} \mathrm{E}\right)$. The altitude is $18.5 \mathrm{~km}$.

and the spectra measured by the spectrometer at the ground indicate that the source is the Sun. The main solar Fraunhofer lines are all clearly identified to coincide with our spectra. Also the atmospheric water vapour absorption bands are identified in the NIR region.

From Fig 3 we also see that the scattered light of the event increased in intensity from the blue to the near infra-red. The maximum intensity per wavelength unit varies from $70 \mathrm{R} / \AA$ for $\lambda$ gn the [4820-4920] $\AA$ range, $120 \mathrm{R} / \AA$ for $\lambda$ in the [6280-6620] $\AA$ range, $375 \mathrm{R} / \AA \AA$ for $\lambda$ in the [7250-7450] $\AA$
Table 1. Estimated emission height profile at $800 \mathrm{~nm}$ from the OSIRIS instrument on the Odin satellite, orbit \#9726, 6 December 2002.

\begin{tabular}{cc}
\hline Altitude $[\mathrm{km}]$ & Intensity $(800 \mathrm{~nm})[\mathrm{R} / \AA]$ \\
\hline 26 & 40 \\
23 & 90 \\
18 & 320 \\
15 & 250 \\
10 & 250 \\
6 & 230 \\
\hline
\end{tabular}

range, and up to $1.5 \mathrm{kR} / \AA$ for $\lambda$ in the [8240-8570] $\AA$ range. Note that normal background values are in the range 5$30 \mathrm{R} / \AA$.

\section{Spaceborne observations}

To our knowledge, the OSIRIS instrument on board the Odin satellite was the only space-borne instrument that where able to detect any signals from the 6 December 2002 event. The OSIRIS instrument measure spectral radiance height profiles over 280 to $800 \mathrm{~nm}$ from 5 to $70 \mathrm{~km}$ altitude at the Earth's limb (Lloyd et al., 2005).

During orbit \#9726, the Odin spacecraft measured enhanced red spectra from 12:47:09 to 12:47:43 UT. The spectra originated from an area that spanned from $\left(72.67^{\circ} \mathrm{N}\right.$, $\left.53.47^{\circ} \mathrm{E}\right)$ to $\left(74.43^{\circ} \mathrm{N}, 49.94^{\circ} \mathrm{E}\right)$, which is slightly southeast of Longyearbyen. Figure 4 shows a typical spectrum of the event as seen by the OSIRIS instrument. The spectra show $\mathrm{O}_{2}$ A-band absorption at $762 \mathrm{~nm}$. The water vapour absorption feature is evident at $\sim 730 \mathrm{~nm}$ and the $\mathrm{O}_{2} \mathrm{~B}$-band at $\sim 690 \mathrm{~nm}$ is also evident. Note that the A-band absorption is unreliable as the background signal that is subtracted (mostly dark current) does have significant A-band emissions.

The spatial resolution of OSIRIS is about $1 \mathrm{~km}$ in altitude by $30 \mathrm{~km}$ parallel to the Earth's surface. The data shown in Table 1 are the radiances observed at $800 \mathrm{~nm}$ by OSIRIS in the limb at different tangent altitudes. Each observation corresponds to the integral of the volume emission rate along the line of sight. Since OSIRIS is outside the Earth's atmosphere, each line of sight observation must have contributions from all altitudes above the tangent point.

When OSIRIS is looking at altitudes above a layer $(26 \mathrm{~km}$ and above), there is no contribution from the layer to the integrated line of sight signal. However when OSIRIS looks at tangent altitudes below or within the layer, then there is always some contribution to the observed radiance from the parts of the layer that are above the tangent point. The result is that you do not expect to see a sharp peak in intensity at the layer altitude but rather a steady decline in intensity as you look at altitudes below or within the layer. 


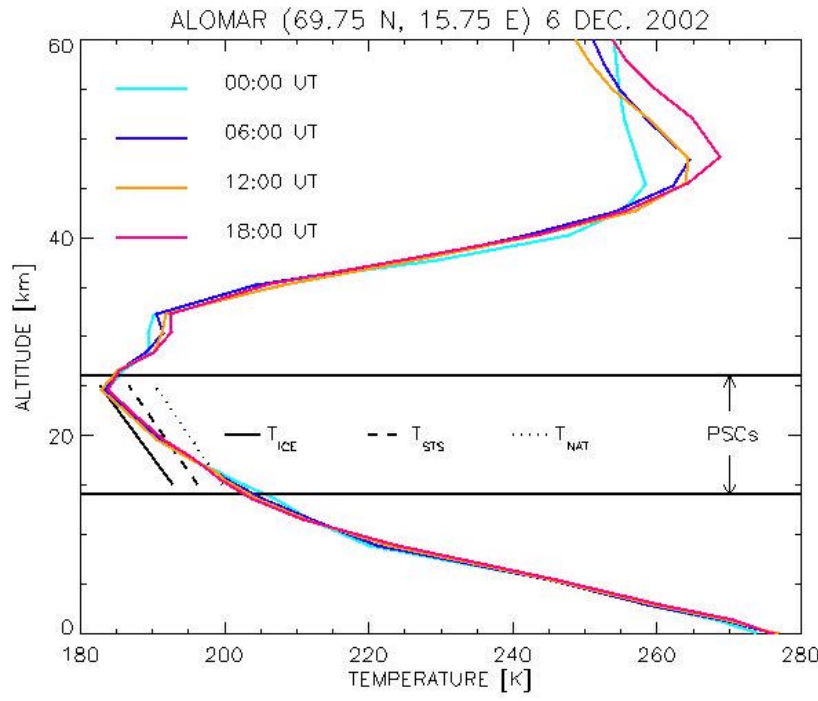

Fig. 5. Temperature height profiles from the European Centre for Medium Range Weather Forecast (ECMWF). The location is Andenes on Andøya, Norway (69.75 N, 15.75 E). Date is 6 December 2002. The profiles are colour coded according to a $6 \mathrm{~h}$ sample period. Also shown are the height region where PSCs are formed together with the 5 ppmv $\mathrm{H}_{2} \mathrm{O}$ threshold temperatures for NAT (dotted line), STS (dashed line) and ice (solid line).

Table 2. Different types of Polar Stratospheric Clouds (PSCs) according to composition (Voigt et al., 2000; Schreiner et al., 1999).

\begin{tabular}{ll}
\hline Type & Composition \\
\hline PSC Ia & $\begin{array}{l}\text { Solid Nitric acid Trihydrate; Nitric acid Dihydrate } \\
\text { (NAT; NAD) particles } \sim 1 \mu \text { m diameter. }\end{array}$ \\
PSC Ib & $\begin{array}{l}\text { Liquid ternary } \mathrm{H}_{2} \mathrm{O} / \mathrm{HNO}_{3} / \mathrm{H}_{2} \mathrm{SO}_{4} \text { solution } \\
\text { (STS) droplets }\end{array}$ \\
PSC II & Ice particles $\sim 10 \mu$ m diameter \\
\hline
\end{tabular}

The main point to be taken from the OSIRIS radiance profile is that the sharp gradient between 18 and $23 \mathrm{~km}$ clearly locates the highest altitude of the layer within the stratosphere. It definitely excludes mesospheric sources for the red-sky. Whether OSIRIS sees a narrow layer (less than $1 \mathrm{~km}$ ) or a thick slab (many km thick) is not easily answered using the OSIRIS data. In principal it is possible to use the shape of the OSIRIS radiance profile to estimate the thickness of the layer but we have not done this as the data do not have sufficient spatial sampling or signal to noise to justify this analysis. Note that the brightness seen by OSIRIS is of the same order of magnitude (a couple of hundred $\mathrm{R} / \AA$ ) as seen by the instrumentation at the Auroral station in Adventdalen.

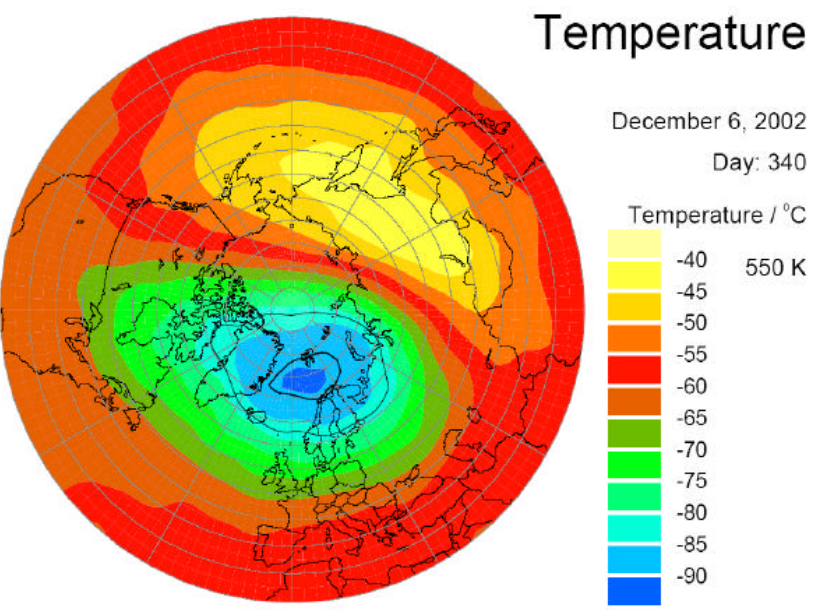

Fig. 6. Northern Hemisphere daily temperatures in the stratosphere from the European Centre for Medium Range Weather Forecast (ECMWF) at a potential temperature of 550K. Date is 6 December 2002. The profiles are colour coded according to temperature distribution.

\section{LIDAR observations}

The source of the red-sky event is apparently located in the Stratosphere. The next interesting question then becomes: What are the temperatures in this region of interest? To answer this question, data from the European Center for Medium Range Weather Forecast (ECMWF) have been obtained. Figure 5 shows the resulting $6 \mathrm{hr}$ temperature height profiles at the Arctic LIDAR Observatory for Middle Atmospheric Research (ALOMAR) $\left(69.75^{\circ} \mathrm{N}, 15.75^{\circ} \mathrm{E}\right)$. The site is about $900 \mathrm{~km}$ South of Longyearbyen. The data show temperatures low enough to form high altitude stratospheric clouds during the period of our event.

It is well known that below a certain threshold temperature, Polar Stratospheric Clouds can form (Poole and McCormick, 1988). There are mainly 3 types of PSCs, distinguished by their particle composition. The different types of PSCs are listed in Table 2.

The threshold temperatures shown in Fig. 5 as a function of height, $\mathrm{T}_{N A T}$ for PSC Ia, $\mathrm{T}_{I C E}$ for PSC II and $\mathrm{T}_{S T S}$ for PSC Ib, were obtained from Muller et al. (2001). Note that typically 5 ppmv $\mathrm{H}_{2} 0$ and 9-10 ppbv $\mathrm{HNO}_{3}$ are assumed as trace gas input for the calculations of PSC threshold temperatures (Koop et al., 1997; Tabazadeh et al., 2001). The temperature above ALOMAR was indeed low enough for PSCs of type I to be formed on 6 December 2002.

In order to proceed, an analysis of ECMWF data was conducted by P. von der Gathen at AWI to include the geographical extent of temperatures in the Stratosphere for 6 December 2002. Figure 6 shows the temperature distribution in the Stratosphere at a potential temperature of $550 \mathrm{~K}$. Within the plot, two black colored line contours are drawn which include the areas where temperature is below the existence temperature of PSC's type I (outer), or even type II (inner 

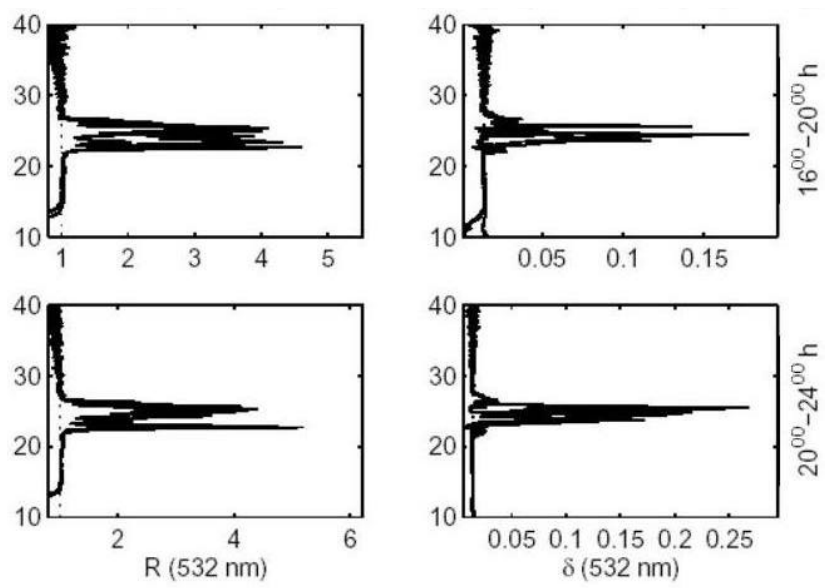

Fig. 7. LIDAR measurements from the Koldewey Station in NyÅlesund on 7 December 2002. The signals are time averaged from 16:00-20:00 UT and 20:00-24:00 UT, respectively. R is the backscatter ratio as a function of altitude $(10-40 \mathrm{~km}) . \delta$ is the corresponding Depolarization. The wavelength is $532 \mathrm{~nm}$.
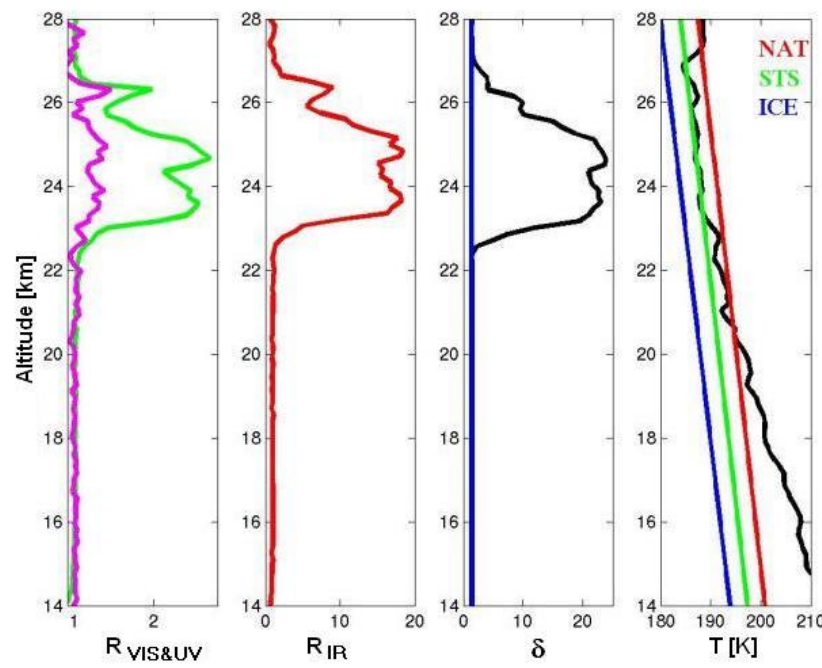

Fig. 8. LIDAR measurements from the Koldewey Station in NyÅlesund on 9 December 2002. The signals are time averaged from 16:00-24:00 UT. R is the backscatter ratio as a function of altitude and wavelength (532 nm VIS (Green); $353 \mathrm{~nm}$ UV (Indigo) and $1064 \mathrm{~nm}$ IR (Red)). $\delta$ is the Depolarization values. The deduced temperature (black line) is plotted together with PSC threshold temperatures for NAT (blue line), STS (green line) and ice (blue line).

curve). As expected, the main PSC II area is exactly between Svalbard and Scandinavia.

The above promising results led us to inquire whether the LIDAR system at the Koldewey Station in Ny-Ålesund operated by the Alfred Wegner Institute (AWI) detected anything unusual during the event. Unfortunately, the weather in NyÅlesund was completely overcast on 6 December 2002. The LIDAR was on the other hand in operation both on 7 and 9 of December 2002. Figures 7 and 8 show the obtained mea- sured height profiles obtained from Ny-Ålesund. The optical characteristics of the data, high backscatter ratios and depolarization values, provide us the evidence for the occurrence of PSCs within this area of cold temperatures (Toon et al., 2000).

The PSC layer above Ny-Ålesund was located in the altitude range $23-27 \mathrm{~km}$. The PSC contained crystalline particles (seen from the increased depolarization values) and was a fairly thick cloud, in vertical extension, as well as in the backscatter ratio values. Together with the OSIRIS observations and those from ALOMAR, this strongly support that a PSC was present in the right place, time, and altitude to produce scattered light, which was observed in Longyearbyen.

The sky was cloudy over Longyearbyen on 7 to 9 of December 2002. This is the main reason why there was no observed red-sky during this period from the Auroral Station in Adventdalen. It is also worth mentioning that the event was observed by the naked eye from North-East of Svalbard, Kinnvika $\left(80^{\circ} \mathrm{N}, 18^{\circ} \mathrm{E}\right)$, on 5, 6, and 7 December 2002 (Trinks, 2003). From this location the southeast horizon is not obscured by any high mountains, except for the glazier Vestfonna blocking the field of view only $1.1 \mathrm{deg}$.

\section{Summary observations}

The data collected during the 6 December 2002 event from

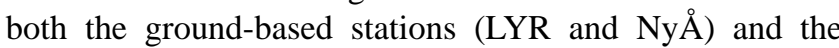
Odin/OSIRIS space borne instrument add the following information.

Ground-based observations

1. The event was symmetric around solar noon. Near winter solstice the Sun is at solar noon 10.7 deg below the horizon in Longyearbyen. If we assume the event is an emitting layer as a result of the direct and immediate action of sun light, then we can apply the method as described by Chamberlain (1961) to calculate the altitudes as a function of view angle from zenith. Figure 9 shows a two-dimensional reproduction of the geometry of twilight scattering used by Chamberlain (1961). The solar depression angle at 10:00 UT was $\alpha=10.9^{\circ}$ and the azimuth angle of observation was close to $\Delta \phi \mathrm{g} 21^{\circ}$ southeast. If we include refraction and use a screening height of $\mathrm{h}_{0}=12 \mathrm{~km}$, the actual shadow heights $\mathrm{z}_{0}$ ranged from 125 to $35 \mathrm{~km}$ for view angles, $\zeta \mathrm{s}$ between zenith down to the horizon, respectively. The solid Earth shadow was $31 \mathrm{~km}$ at the horizon.

2. No abnormal low temperatures in the upper mesosphere were detected by the spectrometers. The hourly average temperature both prior and after the event was close to $200 \mathrm{~K}$. This rules out that the event was caused by sunlit Noctilucent Clouds (NLC)-formed when the temperature is close to the summer minimum $(\sim 120 \mathrm{~K})$. The temperatures were calculated using the $\mathrm{OH}(6-2)$ band of airglow (cf. Sigernes et al., 2003). 
3. It is clear from the spectrometer data that the source of the illumination is the sun. Fraunhofer lines are easily identified in the spectra recorded in zenith from both ground-based sites. Also, since the event is gradually increasing in intensity from weak blue to strong near infra-red spectra, the solar rays must have suffered absorption and scattering by a target above the screening height. In addition, air itself scatters the rays (Rayleigh scattering). It is only the deep red component of the visible solar spectrum that is left. Next, after the initial rays are reflected / scattered, they re-enter the lower atmosphere. Once more the rays suffer scattering. In addition, the ground albedo is high due to the snow covered ground. As a net result, the instruments detect light that has been reflected and scattered several times before it finally enters the narrow $5^{\circ}$ field of view in zenith.

4. The effect of scattering and albedo is also seen in the in Fig. 1. It is hard to identify any target or structure in the image. It is only the scattered component of the light we detect. The intensities of the MSP are still quite high for view angles greater than $175^{\circ}$, even though the mountains and the low cloud cover blocks the direct line of sight to the event! As a consequence, we believe that what we detect is scattered light from below the horizon.

5. It then follows, since the solid Earth shadow was at most $31 \mathrm{~km}$ high as seen towards the horizon, that the projection onto the celestial sphere between the solid Earth shadow line and the stations horizontal line of sight is located about $625 \mathrm{~km}$ southeast. This means that the target that is illuminated by the sun is close to or below $75^{\circ}$ North. The target must be located in the Stratosphere.

Spaceborne observations

6. The OSIRIS spectra have clear signatures of $\mathrm{O}_{2} \mathrm{~B}$-band and water vapour absorptions. These signatures are often seen by OSIRIS under twilight conditions and indicate the rays of light have travelled a substantial distance through the troposphere. It is therefore highly unlikely that the source of red light could be anything but the sun. The rays of light have also gone close to the ground.

7. OSIRIS did not detect any signal from above $26 \mathrm{~km}$. This means that we can rule out the possibility of any extended high altitude upper mesospheric source. The lack of signal above $26 \mathrm{~km}$ rules out the possibility of a very bright, very localized, high altitude source.

8. The deduced height profile shows that the event is a stratospheric phenomenon. If the red- sky was created by a very bright, high altitude scattering source, then it should be expected that the height profile should follow the atmospheric density as the vast majority of scattered signal detected by OSIRIS would be Rayleigh scattered. This is not seen. Instead the height profile resembles a scattering layer with an upper altitude around

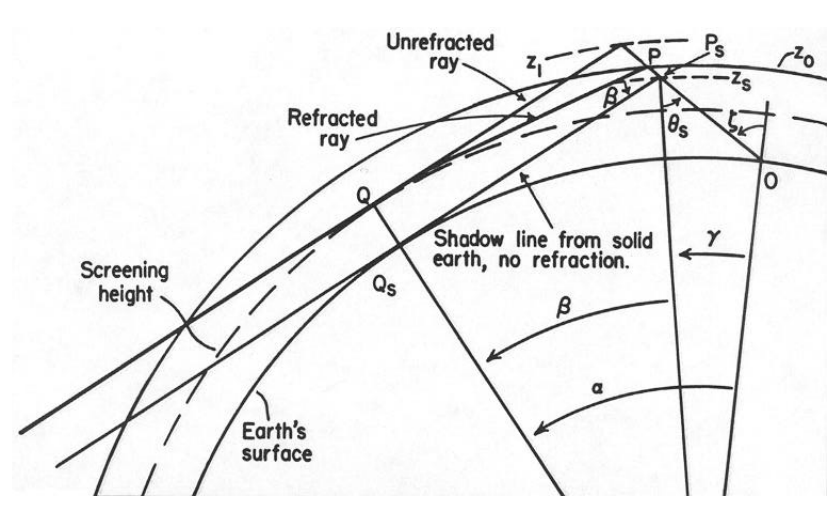

Fig. 9. A reproduction from Chamberlain (1961). Two-dimensional geometry of twilight scattering. The incident ray that passes just above the screening height, $\mathrm{h}_{0}$, intersects the line of sight at height $\mathrm{z}_{0}$, where the lowering of the incident ray by refraction is also included. $z_{0}$ is called the actual shadow height. $\zeta$ is the view angle of the observer, O. $z_{S}$ is the apparent height, the height of the shadow from the Solid Earth with no refraction.

\section{$18-23 \mathrm{~km}$.}

\section{LIDAR observations}

9. The European Centre for Medium Range Weather Forecast (ECMWF) analysis concludes that there is an extended area of low enough temperatures for PSC's to be formed between Scandinavia and Svalbard, consistent with the ground-based observations and the data from OSIRIS.

10. LIDAR measurements from the Koldewey Station in Ny-Ålesund provide evidence for the occurrence of PSCs within this area of cold temperatures from both the 7 and 9 of December 2002.

11. The PSC layer was measured to be in the altitude range 23-27 km, which in vertical extension corresponds to a fairly thick cloud, at most half a pressure scale height.

12. As seen from the increased depolarization values of the LIDARS, the PSC's contained crystalline particles. The low temperatures from the ECMWF even suggest that PSC type II clouds are formed.

Note that the obtained depolarisation values together with the cold stratospheric temperatures rules out the possibility that the event is caused by aerosols as reported by Gerding et al. (2003). It is also worth mentioning that mid-latitude LIDAR observations from Kuehlungsborn $\left(54^{\circ} \mathrm{N}, 12^{\circ} \mathrm{E}\right)$ detected PSC type II conditions in early December, 2002. (Private communication Gerding, IAP).

\section{Analysis of red-sky}

The data-sets, when taken together, suggest that the redsky phenomenon was related to the occurrence of PSC distributed over a relatively large geographical area. First we 

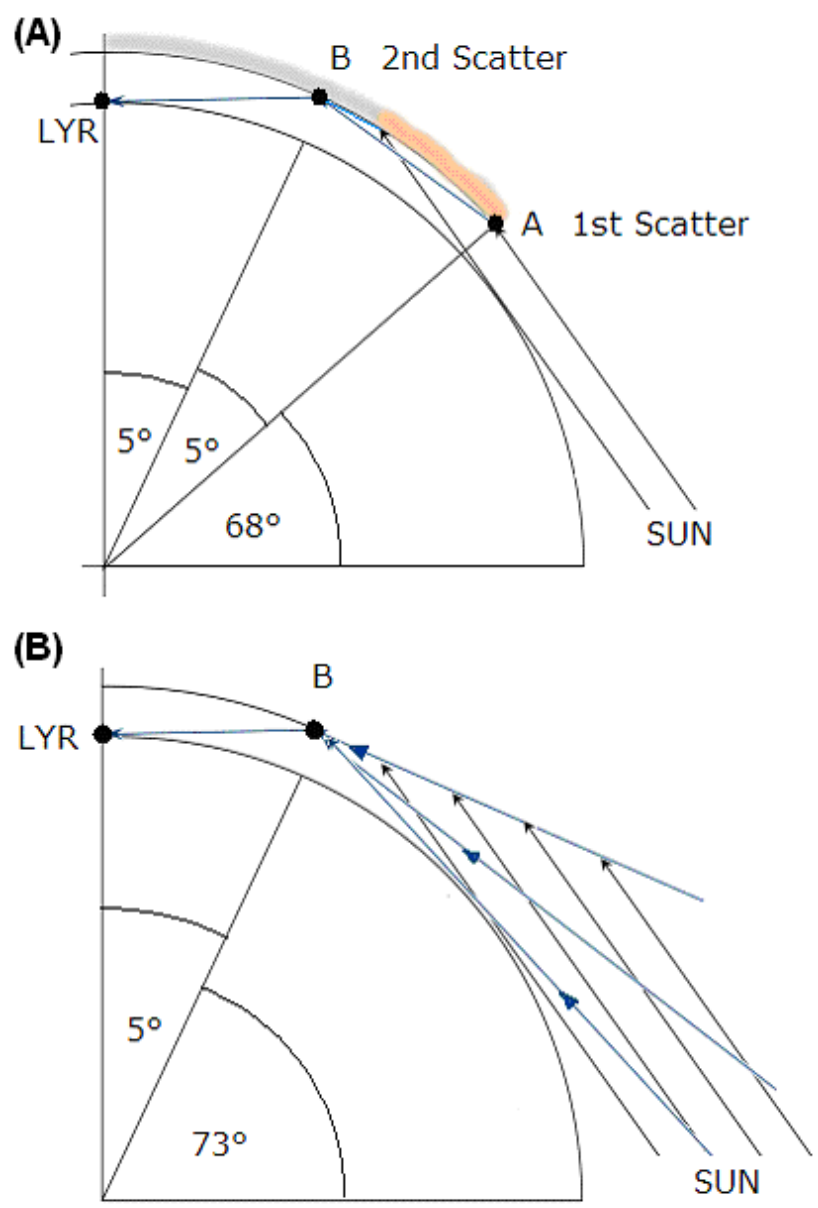

Fig. 10. Two scenarios for the red-sky enigma: (A) PSC Cloud-toCloud scattering mechanism and (B) PSC twilight illumination.

note the obvious facts: it should have been completely dark at LYR as the sun was always $10.5^{\circ}$ or more below the horizon. Second, the presence of solar Fraunhofer and terrestrial absorption features clearly identify the source of the light as sunlight that has passed near to the surface of the Earth.

Since we know that the sunlight was bent around the Earth, we speculate that the mechanism may be one of two possible processes. The first candidate, illustrated in Fig. 10 (Panel A), is PSC cloud-to-cloud scattering where the sunlight is piped around the Earth by a PSC located close to $68^{\circ} \mathrm{N}$, to a second PSC located at $73^{\circ} \mathrm{N}$. It is this second, illuminated PSC that is observed from LYR. The second candidate, illustrated in Fig. 10 (Panel B), is the direct illumination of a PSC located at $73^{\circ} \mathrm{N}$ by Rayleigh scattered twilight. Both of the mechanisms considered here require that PSC occur from $73^{\circ} \mathrm{N}$ to LYR.

The spectral colour of the red-sky is easily explained by the extinction of sunlight by Rayleigh scattering and ozone absorption. Figure 11 shows the modelled spectrum at LYR at 10:00 UT on 6 December 2002 using the cloud-to-cloud scattering process. A detailed description of the radiative transfer model is given by Lloyd et al. (2005). The fig-

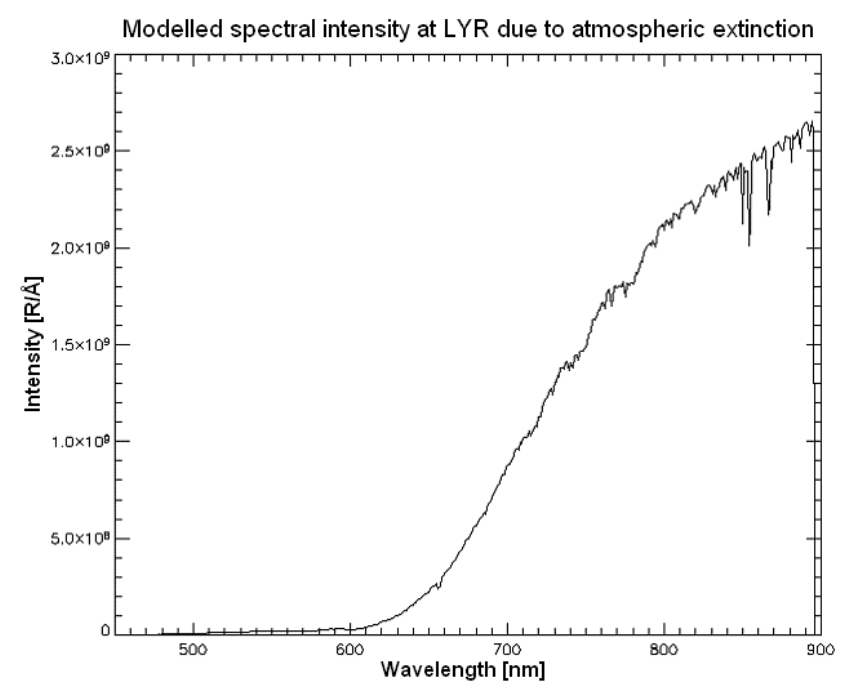

Fig. 11. Modelled spectral intensity of scattered sun light as viewed from Longyearbyen $\left(78^{\circ} \mathrm{N}, 15^{\circ} \mathrm{E}\right)$ 10:00 UT on 6 December 2002.

ure only shows atmospheric extinction along the path length and does not include any calculations for the cloud to cloud scattering cross-sections. The calculation uses the dry air, Rayleigh scattering, cross-sections reported by Bates (1984), the extended MSIS-90 neutral atmosphere (Hedin, 1991), the UGAMP ozone model (Li and Shine, 1995) and solar spectrum by Kurucz et al. (1984). We have not modelled the molecular absorption features due to $\mathrm{O}_{2}$ or $\mathrm{H}_{2} \mathrm{O}$. It is readily evident from Fig. 11 that only sunlight at wavelengths greater than $600 \mathrm{~nm}$ are reaching LYR and the shape of the spectrum seems to be in qualitative agreement with the MSP and spectrograph data. The optical depths implied by Fig. 11 are dominated by the Rayleigh extinction along the path from the PSC at $73^{\circ} \mathrm{N}$ to LYR. Since this path is common to both proposed mechanisms the colour of the spectrum is not very useful for distinguishing between the two mechanisms.

Cloud-to-cloud mechanism

The PSC cloud-to-cloud mechanism derives its inspiration from the fact that the red-sky was observed for $5 \mathrm{~h}$ from 7:30 UT to 12:30 UT. If we assume the red-sky is due to a lower latitude PSC bathed in sunlight then we can immediately calculate that the cloud must between $68^{\circ} \mathrm{N}-69^{\circ} \mathrm{N}$ at $27.5^{\circ} \mathrm{E}$ as this is the only location between $20-25 \mathrm{~km}$ that sees the sun for the $5 \mathrm{~h}$ between 7:30 UT and 12:30 UT. This model is quite appealing in that the bright, lower latitude cloud is southeast of LYR which is where the brightest signal is seen and will naturally produce the brightest signal at 10:00 UT. A crude, order of magnitude, estimate of the signal brightness at LYR can be made by assuming the PSC at A and $\mathrm{B}$ have a vertical extinction of 0.01 which is completely scattered and that to an order of magnitude much of the scattering goes into the forward scatter direction and that the PSC at $68^{\circ} \mathrm{N}$ supports a solid angle of approximately $5.9^{\circ}$ by $5.9^{\circ}$ (or 0.01 steradians) from B. Using these assumptions and 
reading the value at $700 \mathrm{~nm}$ off Fig. 11 we find that the signal at $700 \mathrm{~nm}$ at LYR is $1.0 \times 10^{9} \times 0.01 \times 0.01 \times 0.01=1 \mathrm{kR} / \mathrm{nm}$. Clearly, this value is in order of magnitude agreement with observations and significantly better agreement could be easily achieved by adjusting the various free parameters.

\section{PSC twilight illumination}

The second mechanism is the illumination of a PSC at $73^{\circ} \mathrm{N}$ by a twilight signal. This model is very similar to the Cloudto-Cloud mechanism except that lower latitude PSC is replaced by sunlight which is Rayleigh scattered off the atmosphere. This is a relatively complex calculation which we shall leave for another time but it is sufficient to note that the Rayleigh extinction of the lower atmosphere is comparable, in order of magnitude, to the extinction of the lower latitude PSC in the Cloud to Cloud mechanism and hence can direct comparable amounts of light around the Earth to the PSC at $73^{\circ} \mathrm{N}$.

However the PSC twilight illumination model does have several difficulties. The twilight model will produce the brightest signals very close to the horizon while the maximum signal was observed $12 \mathrm{deg}$ from the horizon. In addition the signal would peak when the sun was due south of the PSC at 10:30 UT while the signal actually peaks half an hour earlier at 10:00 UT. Indeed one would expect the brightest region to follow the sun and steadily move from the southeast through South and into the South-West during the course of the day if the PSC had any longitudinal extent. Hence one has to assume with this model that instrumentation at LYR saw the longitudinal edge of the PSC.

The final problem with the twilight illuminated cloud is that it is probably not a rare enough occurrence. If the redsky only required one PSC just south of LYR during the winter months then you might expect to witness them much more frequently than you actually do.

\section{Concluding remark}

The red-sky observed from Svalbard on 6 December 2002 is identified to be scattered solar light originating from type II Polar Stratospheric Clouds (PSC) located at about $73^{\circ} \mathrm{N}$ South East of Longyearbyen - between Svalbard and Scandinavia. The altitude was $18-23 \mathrm{~km}$ up in the Stratosphere.

Two hypotheses that are able to explain the red-sky phenomenon in terms of sunlight scattered around the Earth are presented. Both models require PSC at latitudes north of $73^{\circ} \mathrm{N}$ which scatter the light into the instrumentation at LYR. The first model, which we feel fits the observations quite well, is PSC cloud to cloud scattering with employs a second lower latitude PSC located at $68^{\circ} \mathrm{N}, 27.5^{\circ} \mathrm{E}$ to scatter direct sunlight to the more northern PSC. The second model involves the twilight illumination of the PSC at $73^{\circ} \mathrm{N}$. Both models are able to direct the correct, order of magnitude, amounts of light but without more information on the actual PSC composition, geographical extent and more complex modelling it is difficult to clearly pick one model over the other. A more detailed model including multiple PSC scattering is given by Lloyd et al. (2005).

Acknowledgements. The Auroral station in Adventdalen is owned by the University of Troms $\emptyset$. The University Courses on Svalbard (UNIS) operates the station. We deeply appreciate the support of the Optical group at the Geophysical Institute, University of Alaska, who owns most of the instruments used in this study. The ECMWF analysis was kindly provided to us by F. Baumgarten at ALOMAR and by P. von der Gathen at AWI.

Topical Editor O. Boucher thanks two referees for their help in evaluating this paper.

\section{References}

Bates, D. R.: Rayleigh scattering in air, Planet. Space Sci., 32(6), 785-790, 1984

Chamberlain, J. W.: Physics of the Aurora and Airglow, Academic Press, New York and London, 1961.

Fastie, W. G.: A small plane grating monochromator, J. Opt. Soc. Am., 42, 641, 1952a.

Fastie, W. G.: Image forming properties of the Ebert monochromator, J. Opt. Soc. Am., 42, 647, 1952 b.

Gerding, M., Baumgarten, G., Blum, U., Thayer, J. P., Fricke, K.H., Neuber, R., and Fiedler, J.: Observations of an unusual midstratospheric aerosol layer in the Arctic: possible sources and implications for polar vortex dynamics, Ann. Geophys., 21, 10571069, 2003,

SRef-ID: 1432-0576/ag/2003-21-1057.

Hauke, T.: Scientific expedition "Life in ice", August 2002-August 2003, Observations at Kinnvika $\left(80^{\circ} 3^{\prime} \mathrm{N}, 18^{\circ} 12^{\prime} \mathrm{E}\right)$ Svalbard, Nordaustland, final report, 1 . October 2003, Technical University of Hamburg-Harburg, Hamburg, Germany, 2003.

Hedin, A. E.: Neutral atmosphere empirical model from the surface to lower exosphere MSIS90, J. Geophys. Res., 96, 1159-1172, 1991.

Koop, T., Carslaw, K. S., and Peter, T.: Thermodynamic stability and phase transitions of PSC particles, Geophys. Res. Lett., 24, 2199-2202, 1997.

Kurucz, R. L., Furenlid, I., Brault, J., and Testerman, L.: Solar Flux Atlas from 296 to $1300 \mathrm{~nm}$, National Solar Observatory Atlas No. 1, 2nd ed., 1984.

Lloyd, N. D., Degenstein, D. A., Sigernes, F., Llewellyn, E. J., and Lorentzen, D. A.: A mechanism for the red-sky enigma: Ducting of sunlight by Polar Stratospheric Clouds, Ann. Geophys., in press, 2005.

Li, D. and Shine, K. P.: A 4-Dimensional Ozone Climatology for UGAMP Models., UGAMP Internal Report No. 35, April 1995, 1995.

Muller, M., Neuber, R., Beyerle, G., Kyro, E., Kivi, R., and Woste, L.: Non-uniform PSC occurrence within the Arctic polar vortex, Geophys. Res. Lett., 28, 4175-7178, 2001.

Pedersen, T.: Mystisk fenomen: Opplyst polarnatt forundrer forskerne, Svalbardposten, no. 49, 54, 2002.

Poole, L. R. and McCormick, M. P.; Airborne lidar observations of Arctic polar stratospheric clouds: Indications of two distinct growth stages, Geophys. Res. Lett., 15, 21-23, 1988.

Schreiner, J., Voigt, C., Kohlmann, A., Arnold, F., Mauersberger, K., and Larsen, N.: Chemical analysis of polar stratospheric cloud particles, Science, 283, 968-970, 1999. 
Sigernes, F., Shumilov, N., Deehr, C. S., Nielsen, K. P., Svenøe, T., and Havnes, O.: The Hydroxyl rotational temperature record from the Auroral Station in Adventdalen, Svalbard $\left(78^{\circ} \mathrm{N}, 15^{\circ} \mathrm{E}\right)$, J. Geophys. Res., 108(A9), 1342, doi:1029/2001JA009023, 2003.

Tabazadeh, A., Jensen, E. J., Toon, O. B., Drdla, K., and Schoeberl, M. R.: Role of the stratospheric polar freezing belt in denitrification, Science, 291, 2591-2594, 2001.
Toon, O. B., Tabazadeh, A., Browell, E. V., and Jordan, J.: Analysis of lidar observations of Arctic polar stratospheric clouds during January 1989. J. Geophys. Res., 105, 20, 589-20, 615, 2000.

Voigt, Ch., Schreiner, J., Kohlmann, A., Zink, P., Mauersberger, K., Larsen, N., Deshler, T., Kröger, C., Rosen, J., Adriani, A., Cairo, F., Di Donfrancesco, D., Viterbini, M., Ovarlez, J., Ovarlez, H., David, Ch., and Dörnbrack, A.: Nitric Acid Trihydrate (NAT) in Polar Stratospheric Clouds, Science, 290, 1756-1758, 2000. 